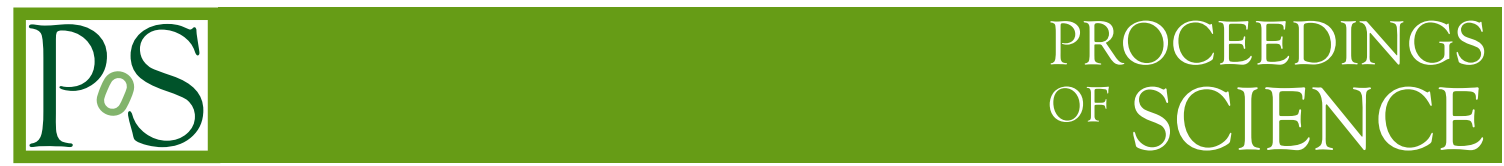

\title{
Recent measurements of top quark properties in CMS
}

\section{Giulia Negro $^{a, *}$ on behalf of the CMS Collaboration}

a Purdue University,

525 Northwestern Ave, West Lafayette, Indiana, US

E-mail: giulia.negro@cern.ch

Measurements of top quark properties using data collected by the CMS experiment are presented. Among them, the latest results on the $t \bar{t}$ spin correlations, the $\mathrm{W}$ boson polarization, the top quark Yukawa coupling, the top quark mass and its running, the top sector of the CKM matrix, and the $t \bar{t}$ forward backward asymmetry will be discussed.

40th International Conference on High Energy physics - ICHEP2020

July 28 - August 6, 2020

Prague, Czech Republic (virtual meeting)

${ }^{*}$ Speaker 


\section{Introduction}

The top quark is the heaviest elementary particle discovered so far. It has an extremely short lifetime $\left(10^{-25} \mathrm{~s}\right)$ which allows us to observe properties of the bare quark, and a large Yukawa coupling to the Higgs boson which makes it important to understand the electroweak symmetry breaking. It is also an ideal candidate for spin measurements because the spin information is preserved in the angular distribution of its decay products. Studying the properties of the top quark provide thus crucial information to test the internal consistency of the Standard Model (SM) and to search for new physics phenomena beyond the SM (BSM). Recent results of the CMS experiment [1] are described in the following Sections.

\section{Spin correlations}

The $t \bar{t}$ spin correlation analysis [2] measures the full spin density production matrix in the dilepton channel using 2016 data at $13 \mathrm{TeV}$. First of all, a direct measurement, which requires the full reconstruction of the $t \bar{t}$ system, is performed measuring angular distributions in the $t \bar{t}$ rest frame, parametrized by the polarization and the spin correlations coefficients. The most precise measurement is given by the opening angle between the two leptons, $\cos \varphi=\hat{\ell}_{1} \cdot \hat{\ell}_{2}$, which has maximal sensitivity to the alignment of the spins of the top quark. Then, also an indirect measurement is performed using observables in the laboratory-frame, such as the angle between the leptons in the transverse plane, $\left|\Delta \phi_{\ell \ell}\right|$, which is experimentally very precise thanks to the excellent resolution of the measured lepton angular distributions. The summary of the spin correlations coefficients, shown in Figure 1, confirms that all the extracted parameters are in close agreement with the SM predictions. The unfolded results are then used to constrain the anomalous couplings shown in Figure 1. They are in agreement with the SM, so, no indication of new physics is observed.

\section{$2.1 \Delta \phi$ distribution}

Both ATLAS [3] and CMS [2] show a tension between data and predictions in the $\Delta \phi$ distribution. Within the LHCtopWG an ATLAS-CMS comparison, the first at $13 \mathrm{TeV}$, is being performed using normalized cross sections at parton level. The agreement between the data and the main MC predictions of ATLAS and CMS, shown in Figure 2, is very good and there is a fair agreement with next-to-next-to-leading order (NNLO) calculations.

\section{W polarization}

The polarization fractions of the $\mathrm{W}$ boson are determined by the V-A structure of the $\mathrm{tWb}$ vertex and follow the unitarity constraint in the SM: $F_{0}+F_{L}+F_{R}=1$, where the longitudinal fraction $\left(F_{0}\right)$ represents $\sim 70 \%$, the left one $\left(F_{L}\right) \sim 30 \%$, and the right one $\left(F_{R}\right) \sim 0 \%$. Predictions of SM can thus be tested with high-precision measurements, while potential new physics processes that can change probability of $\mathrm{W}$ helicity states can be probed by anomalous contributions to the $\mathrm{tWb}$ vertex. To measure the polarization fractions, an angular distribution in the $\mathrm{W}$ boson rest frame is used. To improve the experimental precision, an ATLAS-CMS combination with Run 1 data [5] is performed using the lepton+jets channel analysis for ATLAS and the e/ $\mu+j$ ets channel 

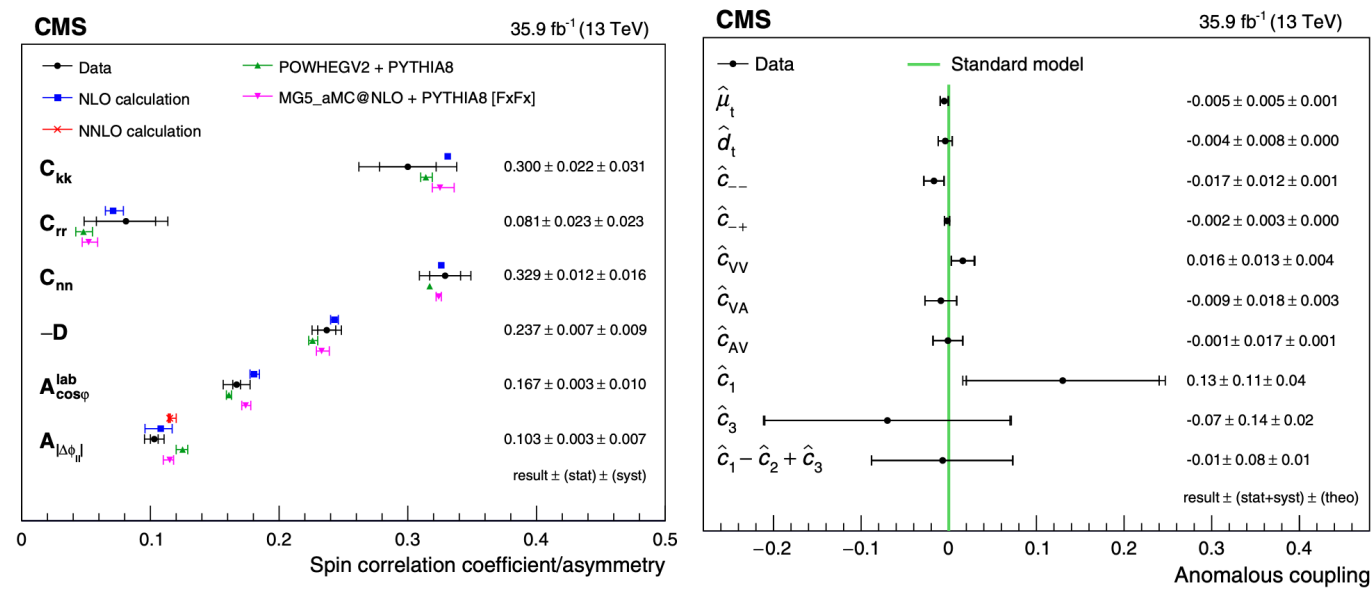

Figure 1: Measured values of the spin correlation coefficients and asymmetries compared to different predictions on the left; measured values of the fitted anomalous couplings on the right. [2]
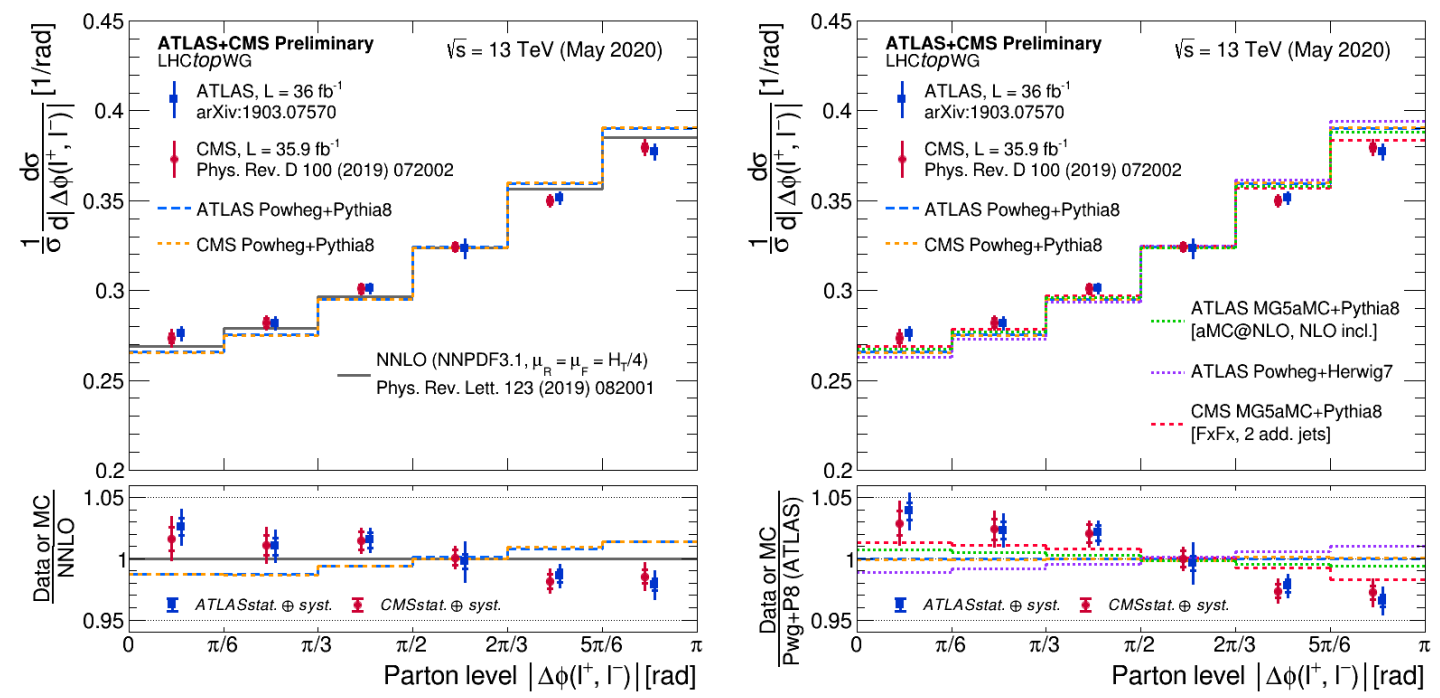

Figure 2: ATLAS and CMS data compared to Powheg+Pythia8 MC from ATLAS and CMS as well as calculations at NNLO on the left, and to different MC simulations on the right. [4]

and single top t-channel analyses for CMS. The results of the combination, shown in Figure 3, are in agreement with the SM at NNLO. Limits on the tWb anomalous couplings are then set and are found to be in agreement with the SM at $95 \%$ confidence level (C.L.).

\section{Top Yukawa coupling}

To extract the top quark Yukawa coupling, weak corrections due to a virtual exchange of a Higgs boson depending on the top-Higgs Yukawa coupling $g_{t}$ are considered. These corrections affect the inclusive $t \bar{t}$ cross section only at a quadratic $\left(\alpha_{S}^{2} \alpha_{w}\right)$ order but may lead to large distorsions of $t \bar{t}$ differential distributions near the production threshold region. Differential cross sections for 


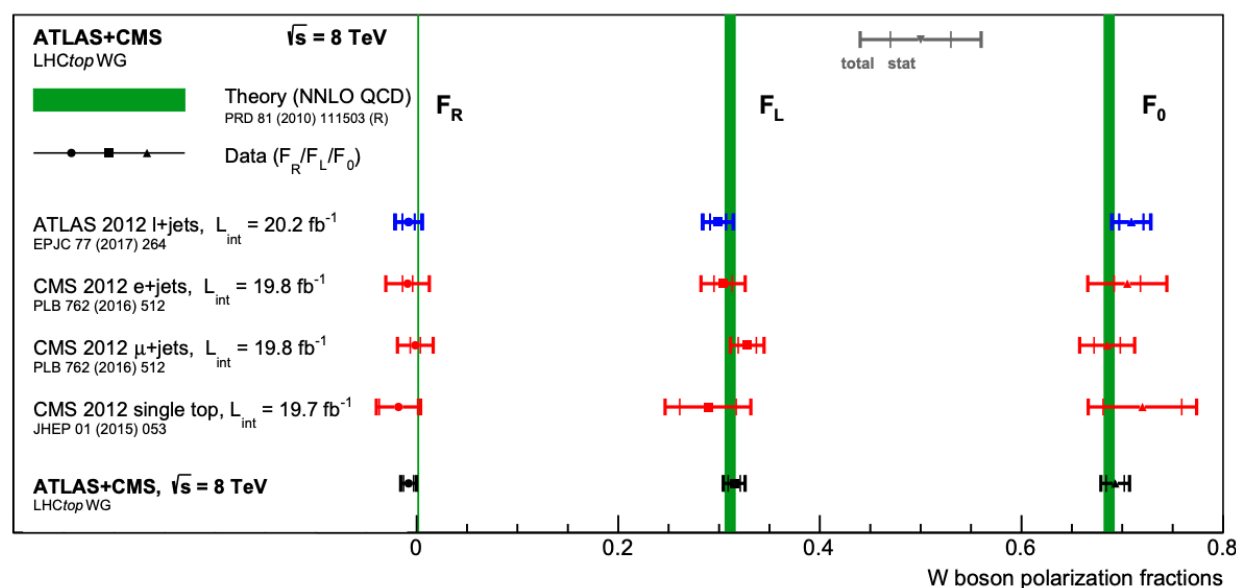

Figure 3: Overview of the four measurements, as well as the results of the combination. [5]

different values of $Y_{t}$, which is the ratio of $g_{t}$ with respect to its SM value $g_{t}^{S M}$, are generated as a function of two variables: the invariant mass $\left(m_{t \bar{t}}\right)$ and the rapidity $\left(\Delta y_{t \bar{t}}\right)$ of the $t \bar{t}$ system. The first analysis to measure the Yukawa coupling with top pair production used 2016 data in the lepton+jets channel [6]. A full kinematic reconstruction was used and a 2D likelihood fit was performed in bins of $m_{t \bar{t}}$ and $\Delta y_{t \bar{t}}$. A new analysis with full Run 2 data [7] measures the Yukawa coupling in the dilepton channel. Even using a partial kinematic reconstruction, an improved sensitivity is achieved using two proxy variables, the invariant mass $\left(m_{b \ell}\right)$ and the rapidity $\left(\Delta y_{b \ell}\right)$ of the b quark and lepton system, for the 2D likelihood fit. The upper limit on the top quark Yukawa coupling is extracted scanning the likelihood fit with respect to $Y_{t}$. The results, respectively $Y_{t}<1.67$ and $Y_{t}<1.62$ at $95 \%$ C.L., show good agreement between the two channels.

\section{Top quark mass measurements}

\subsection{Boosted mass}

The mass of boosted top quarks is measured with 2016 data in the lepton+jet channel [8], where the products of the hadron decay are reconstructed as a single jet. A new jet reconstruction technique, called XCone and giving an excellent resolution on the jet mass, was used for the first time. Normalized $t \bar{t}$ cross sections as a function of the jet mass are then used to extract the top mass: $m_{t}=172.6 \pm 2.5 \mathrm{GeV}$. Its precision of $\sim 0.7 \%$ is impressive considering the boosted topology.

\subsection{Mass from multidifferential distributions}

The mass of the top quark can be measured using multi differential distributions, in particular normalized 3D cross sections as a function of the invariant mass and of the rapidity of $t \bar{t}$, and of the number of extra jets using 2016 data in the dilepton channel [9]. To extract $\alpha_{S}$ and $m_{t}$ in the pole mass scheme $\left(m_{t}^{\text {pole }}\right)$, these cross sections are compared to NLO predictions with different PDF sets and then a simultaneous fit to PDF, $\alpha_{S}$, and $m_{t}^{\text {pole }}$ is performed adding deep inelastic scattering data from HERA. The results are: $\alpha_{S}\left(m_{Z}\right)=0.1135_{-0.0017}^{+0.0021}, m_{t}^{\text {pole }}=170.5 \pm 0.8 \mathrm{GeV}$. The result on $m_{t}^{\text {pole }}$, with a precision below $0.5 \%$, is the most precise one from a single analysis. 


\begin{tabular}{cccc} 
PDF set & $\alpha_{S}\left(m_{Z}\right)$ & $m_{t}\left(m_{t}\right)[\mathrm{GeV}]$ & $m_{t}^{\text {pole }}[\mathrm{GeV}]$ \\
\hline ABMP16 & $0.1139 \pm 0.0023_{-0.0001}^{+0.0014}$ & $161.6 \pm 1.6_{-1.0}^{+0.1}$ & $169.9 \pm 1.8_{-1.2}^{+0.8}$ \\
NNPDF3.1 & $0.1140 \pm 0.0033_{-0.0002}^{+0.0021}$ & $164.5 \pm 1.6_{-1.0}^{+0.1}$ & $173.2 \pm 1.9_{-1.3}^{+0.9}$ \\
CT14 & $0.1148 \pm 0.0032_{-0.00002}^{+0.0018}$ & $165.0 \pm 1.8_{-1.0}^{+0.1}$ & $173.7 \pm 2.0_{-1.4}^{+0.9}$ \\
MMHT14 & $0.1151 \pm 0.0035_{-0.0002}^{+0.0020}$ & $164.9 \pm 1.8_{-1.1}^{+0.1}$ & $173.6 \pm 1.9_{-1.4}^{+0.9}$
\end{tabular}

Table 1: Values of $\alpha_{S}\left(m_{Z}\right), m_{t}\left(m_{t}\right)$, and $m_{t}^{\text {pole }}$ for different PDF sets. The first uncertainty is (fit + PDF), the second one is (scale). [10]

\subsection{Mass from inclusive measurement}

The top quark mass is measured from the inclusive cross section in the e $\mu$ channel with 2016 data [10], where a simultaneous fit of $\sigma_{t \bar{t}}$ and $m_{t}^{M C}$ is performed to extract $\alpha_{S}\left(m_{Z}\right)$ and $m_{t}$ in the minimal subtraction renormalization scheme, and $m_{t}^{\text {pole }}$. The values obtained for different PDF sets are consistent within each other, as shown in Table 1.

\subsection{Running of top mass}

For the first time the running of the top mass was measured with 2016 data [11]. It is extracted comparing differential cross sections measured as a function of $m_{t \bar{t}}$ in the e $\mu$ channel to NLO predictions with different top masses. A maximum likelihood fit to multi-differential distributions is performed to do a simultaneous measurement of $m_{t}^{M C}$ and of the differential cross section of $t \bar{t}$ as a function of $m_{t \bar{t}}$. The mass running is found in agreement with the prediction of the renormalization group equation within $1.1 \sigma$ and the no-running scenario is excluded at above $95 \%$ C.L..

\section{Top CKM elements}

To extract the top CKM matrix elements, a direct model-independent measurement is performed for the first time in single top t-channel events with 2016 data [12]. Processes directly sensitive to $\left|V_{t b}\right|,\left|V_{t d}\right|$, and $\left|V_{t s}\right|$ are considered at both the production and decay vertices of the top quark. Three categories, each enriched with different signal and background processes, are defined. A BDT discriminant is trained for each category to separate signal and background, and then is used in a simultaneous fit to the three categories to discriminate between the different signals. The CKM matrix elements are extracted by the measured single top t-channel cross sections times their branching ratios compared to their expected values. Assuming the CKM unitarity in the SM, the following limits are obtained at 95\% C.L.: $\left|V_{t b}\right|>0.970$ and $\left|V_{t d}\right|^{2}+\left|V_{t s}\right|^{2}<0.057$. Then also two possible BSM scenarios are investigated. All results are consistent with each other and represent the best determination with respect to the latest measurements of single top quark in Run 2 .

\section{Forward-Backward asymmetry}

The Forward-Backward asymmetry $\left(A_{F B}\right)$ in $t \bar{t}$ production is given by NLO interference terms between $q \bar{q}$ initial states and is measured, together with the anomalous chromoelectric $\left(d_{t}\right)$ and chromomagnetic $\left(\mu_{t}\right)$ dipole moments, by the parametrization of angular distributions of produced $t \bar{t}$ pairs. The measurement is performed in the lepton+jets channel with 2016 data in boosted and 
resolved topologies [13]. A multi-dimensional template fit is performed for each topology and their linear combination is used in a simultaneous fit to the observed differential cross sections to extract the parameters: $A_{F B}^{(1)}=0.048_{-0.087}^{+0.095}(\text { stat })_{-0.029}^{+0.020}($ syst $), \mu_{t}=-0.024_{-0.009}^{+0.013}(\text { stat })_{-0.011}^{+0.016}($ syst $)$, and $\left|d_{t}\right|<0.03$ at $95 \%$ C.L.. Their values, measured for the firs time at LHC, are consistent with the SM expectations and in good agreement with previous measurements.

\section{Conclusions}

A lot of analyses with new Run 2 data and also with new channels and techniques has been performed to investigate the top quark properties, several of them probed for the first time. The huge number of top quark events produced allows us to reach an increased precision useful to understand better the top quark properties and to constrain new physics.

\section{References}

[1] CMS Collaboration, JINST 3 (2008), S08004, doi:10.1088/1748-0221/3/08/S08004

[2] CMS Collaboration, Phys. Rev. D 100 (2019) no.7, 072002, doi:10.1103/PhysRevD.100. 072002, arXiv:1907.03729 [hep-ex]

[3] ATLAS Collaboration, Eur. Phys. J. C 80 (2020) no.8, 754. doi:10.1140/epjc/s10052-0208181-6. arXiv:1903.07570 [hep-ex]

[4] https://twiki.cern.ch/twiki/bin/view/LHCPhysics/LHCTopWGSummaryPlots

[5] CMS Collaboration, JHEP 08 (2020) no.08, 051, doi:10.1007/JHEP08(2020)051, arXiv:2005.03799 [hep-ex]

[6] CMS Collaboration, Phys. Rev. D 100 (2019) no.7, 072007, doi:10.1103/PhysRevD.100. 072007, arXiv:1907.01590 [hep-ex]

[7] CMS Collaboration, arXiv:2009.07123 [hep-ex]

[8] CMS Collaboration, Phys. Rev. Lett. 124 (2020) no.20, 202001, doi:10.1103/PhysRevLett.124. 202001, arXiv:1911.03800 [hep-ex]

[9] CMS Collaboration, Eur. Phys. J. C 80 (2020) no.7, 658, doi:10.1140/epjc/s10052-020-79177, arXiv: 1904.05237 [hep-ex]

[10] CMS Collaboration, Eur. Phys. J. C 79 (2019) no.5, 368, doi:10.1140/epjc/s10052-019-68638, arXiv:1812.10505 [hep-ex]

[11] CMS Collaboration, Phys. Lett. B 803 (2020), 135263, doi:10.1016/j.physletb.2020.135263, arXiv: 1909.09193 [hep-ex]

[12] CMS Collaboration, Phys. Lett. B 808 (2020), 135609, doi:10.1016/j.physletb.2020.135609, arXiv:2004.12181 [hep-ex]

[13] CMS Collaboration, JHEP 06 (2020), 146, doi:10.1007/JHEP06(2020)146, arXiv:1912.09540 [hep-ex] 